\title{
Syncope in Acute Pulmonary Embolism. Importance of Early Recognition of Recurrent Syncope as a Manifestation of Acute Bilateral Pulmonary Embolism.
}

\author{
Khalid Sawalha ${ }^{1}$, Shoaib Khan ${ }^{1}$, Bryan Haung ${ }^{1}$, and Naveed Younis ${ }^{1}$ \\ ${ }^{1}$ White River Health System Inc
}

January 20, 2021

\begin{abstract}
A 43-year-old female patient presented with large bilateral pulmonary emboli. During patient's stay, a fleeting episodes of bradycardia and hypotension with drop in systolic pressure over $40 \mathrm{mmHg}$ on slight movements were noted.Being in a rural hospital, patient was transferred in which following that she was pronounced dead relating her presentation.
\end{abstract}

\section{Introduction}

Acute pulmonary embolism (PE) is a form of venous thromboembolism (VTE) that is common and sometimes fatal. The clinical presentation of $\mathrm{PE}$ is variable and often nonspecific making the diagnosis challenging. Recurrent syncope is a rare presentation

of pulmonary embolism that is estimated to occur in $10 \%$ of cases.[1] It can be associated with quick deterioration and poor prognosis. Early recognition of syncope as a mechanism of pulmonary embolism is crucial as the 30-day mortality is significantly higher in these patients. [2] Hence, intervention and early treatment are critical to reduce mortality. We present this case to emphasize the importance of early thrombolytic therapy in patients presenting with syncope-associated pulmonary embolism. 


\section{Case presentation}

A 43-year-old female initially presented to the emergency department with shortness of

breath and syncope. She has a history of superficial DVTs, nicotine dependency, and morbid obesity. On presentation, she was alert and oriented with normal neurological findings. She had an elevated heart rate of over 120 with an oxygen saturation of $90 \%$ on 2L NC and was hemodynamically stable. Her labs were significant for leukocytosis, an elevated D-Dimer of 2.85ug/mL, BNP $17400 \mathrm{pg} / \mathrm{mL}$, and an initial troponin of $0.063 \mathrm{ng} / \mathrm{mL}$. An emergent chest-CT was performed which revealed large bilateral pulmonary emboli (Figure 1). The patient was subsequently started on full-dose Lovenox and sent to the progressive care unit for further evaluation and management. Overnight, the patient was noted to have an episode of syncope that was associated with cyanosis, tremors, and diaphoresis. At that time, her blood pressure was stable at 130/80 as she remained mildly lethargic. As the patient did not meet formal indications for systemic tissue plasminogen activator (tPA), she was immediately transferred to the ICU for further evaluation and closer monitoring of her symptoms. During the day, an

echocardiogram was performed which revealed a severely dilated right atrium and

ventricle with a right ventricular systolic pressure of $66.2 \mathrm{mmHg}$ (Figure 2). The patient had fleeting episodes of bradycardia and hypotension with a noted drop of over $40 \mathrm{mmHg}$ in her systolic pressure on slight movements. Given her rapid decompensation and being in rural setting with limited resources, the patient was immediately transferred to a facility capable of performing intervention catheter-directed thrombolysis for treatment. Following the transfer, the patient was pronounced deceased due to complications related to her condition.

\section{Discussion}

Pulmonary embolus (PE) refers to the obstruction of the pulmonary artery or one of its branches by material such as thrombus, tumor, air, or fat that originated elsewhere in the body. It can be acute, subacute and chronic based on the timeline of its presentation. It is also classified based on the presence or absence of hemodynamic stability, which is associated with right ventricular strain which is also called massive or high-risk PE.

PE has a wide variety of presenting features, ranging from no symptoms to shock or sudden death. The most common presenting symptom is dyspnea followed by pleuritic chest pain, cough, and symptoms of deep venous thrombosis such as lower extremity pain, swelling and tenderness. Hemoptysis is an unusual presenting symptom. Rarely do patients present with shock, arrhythmia, or syncope. Diagnosis usually starts with a high index of clinical suspicion along with elevated D-Dimer and imaging includes computed tomographic pulmonary angiography and less commonly, ventilation perfusion scanning or other imaging modalities. For patients who are hemodynamically unstable and in whom definitive imaging is unsafe, bedside echocardiography or venous compression ultrasound may be used to obtain a presumptive diagnosis of PE to justify the administration of potentially life-saving therapies such as TPA.

In this case, the patient presented to a rural hospital with syncope with subsequent testing showing large bilateral pulmonary embolism. Hence, she was started on full dose Lovenox. Despite having normal vital signs and being on anticoagulation, she deteriorated overnight with recurrent episodes of syncope and hypotension manifested by drops in systolic blood pressure to more than $40 \mathrm{mmhg}$. Due to limited abilities in a rural setting, the decision was made to transfer to a facility capable of catheter directed thrombolysis. Unfortunately, following her transfer she was pronounced dead.

Therefore, the distinction between hemodynamically stable and unstable PE is important because patients with hemodynamically unstable PE are more likely to die from obstructive shock as a result of severe right 
ventricular failure. Syncope associated pulmonary embolism can be fatal and attributed to rapidly decreasing cardiac output if not treated emergently.

\section{Conclusion}

Syncope-associated pulmonary embolism is a medical emergency that can be associated with poor prognosis and rapid decompensation. With a 90-day mortality rate of $17.4 \%$. [3] pulmonary embolism requires heightened clinical suspicion and immediate therapy to prevent fatal pulmonary and cardiac complications. While current guidelines indicate that systemic and catheter-directed thrombolysis are reserved for those with hemodynamic instability, those presenting with recurrent syncope may predict rapid decompensation and may also benefit from early thrombolytic therapy or mechanical thrombectomy if contraindications exist.

\section{References}

1. Demircan A, Aygencel G, Keles A, et al. Pulmonary embolism presenting as syncope: a case report. $J$ Med Case Rep . 2009;3:7440.

2. Roncon L et al. Impact of syncope and pre-syncope on short-term mortality in patients with acute pulmonary embolism. Eur J Intern Med 2018 Apr 11.

3. 1: Altınsoy, Bülent, et al. "Syncope as a presentation of acute pulmonary embolism." Therapeutics and clinical risk management 12 (2016):1023.

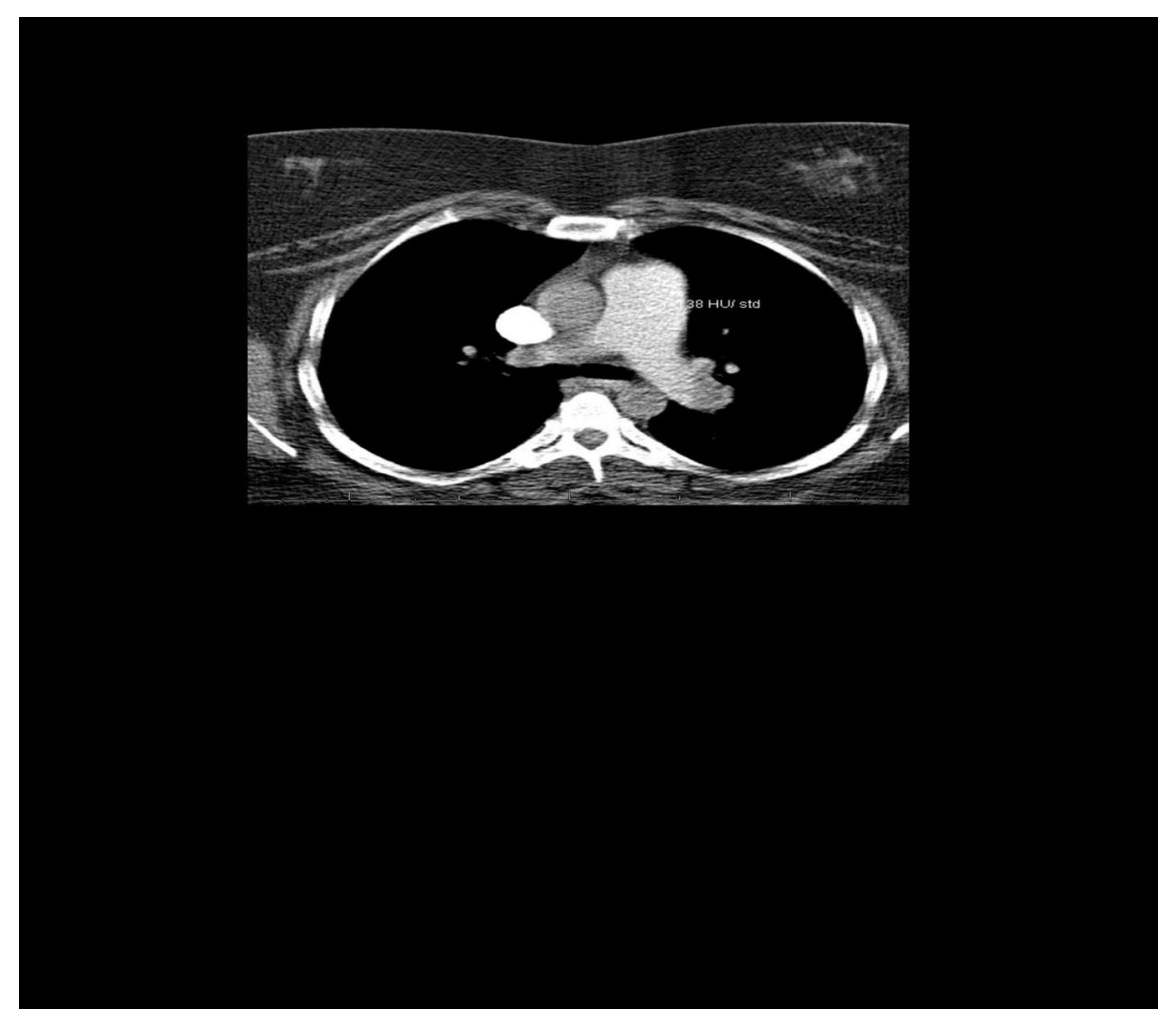




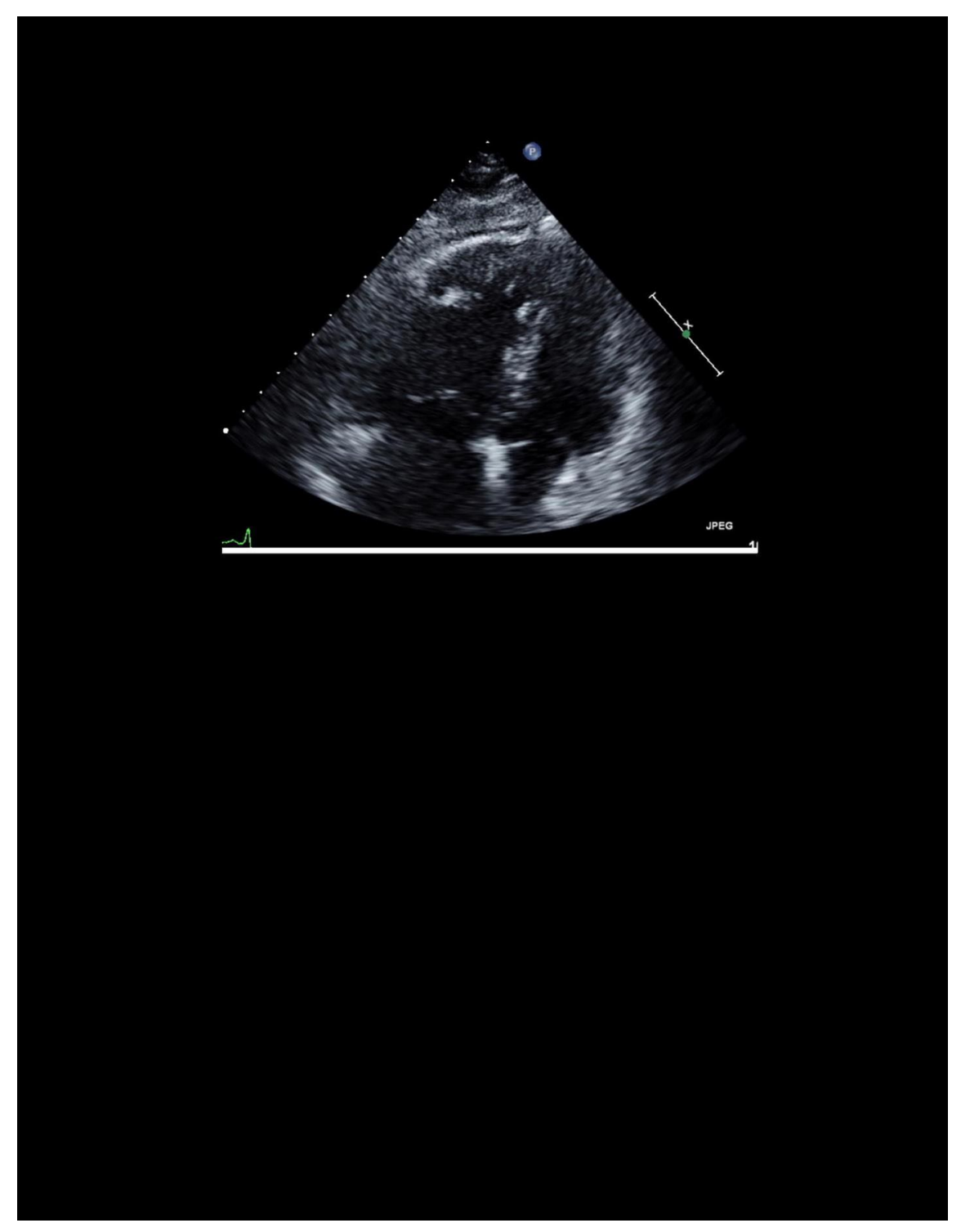

